University of New Mexico

UNM Digital Repository

Mathematics and Statistics Faculty and Staff

Publications

Academic Department Resources

2014

COMMENTS ON THE PAPER “AN ALTERNATIVE COMBINATION

RULE FOR EVIDENTIAL REASONING" BY SEBBAK et al., PUBLISHED IN FUSION 2014 CONFERENCE, SALAMANCA, SPAIN, JULY 2014

Florentin Smarandache

Jean Dezert

Arnaud Martin

Follow this and additional works at: https://digitalrepository.unm.edu/math_fsp

Part of the Control Theory Commons, Dynamic Systems Commons, Logic and Foundations Commons, and the Other Mathematics Commons 


\title{
COMMENTS ON THE PAPER "AN ALTERNATIVE COMBINATION RULE FOR EVIDENTIAL REASONING" BY SEBBAK et al., PUBLISHED IN FUSION 2014 CONFERENCE, SALAMANCA, SPAIN, JULY 2014
}

\author{
Florentin Smarandache, \\ University of New Mexico, Gallup, NM 87301, USA, \\ E-mail: smarand@unm.edu \\ Jean Dezert, \\ The French Aerospace Lab, ONERA/DTIM/EVF, Chemin de la Hunière, 91761 \\ Palaiseau Cedex, France, \\ E-mail: jean.dezert@onera.fr \\ Arnaud Martin, \\ Université de Rennes 1 / IUT de Lannion, UMR 6074 IRISA, \\ Rue Edouard Branly, BP 30219, 22302 Lannion cedex, France, \\ E-mail: Arnaud.Martin@univ-rennes1.fr
}

Received on 30 July 2014: Accepted on 21 October 2014

\begin{abstract}
In this note we want to show that the PCR6 fusion rule works and redistributes the conflicting mass properly, contrarily to the authors' assertion that "the focal element $\left\{v_{3}\right\}$ absorbs almost all of the conflicting mass (the majority)." We also question the validity of the new CREC rule of combination presented by the authors.
\end{abstract}

Keywords: Belief function; PCR6 rule; PCR5 rule; CREC rule.

\section{INTRODUCTION.}

Recently in [1], Sebbak et al. consider the following Example 2, where the frame of discernment is

$\Theta=\left\{v_{1}, v_{2}, v_{3}\right\}$, in Shafer's model (i.e. all intersections of the elements in $\Theta$ are empty), and three sources of information characterized by the basic belief assignments (BBA) $m_{1}, m_{2}$, and $m_{3}$ : 


$\begin{array}{llll} & v_{1} & v_{3} & \Theta \\ m_{1} & 0.8 & 0 & 0.2 \\ m_{2} & 0.1 & 0.9 & 0 \\ m_{3} & 0.4 & 0 & 0.6\end{array}$

We verified by hand and with MatLab the PCR6 fusion of these BBAs and the authors correctly computed PCR6 by combining these three sources altogether, and they got the correct result:

$m_{123 \mid \operatorname{PCR} 6}\left(v_{1}\right)=0.4340, m_{123 \mid P C R 6}\left(v_{3}\right)=0.4437$, and $m_{123 \mid \operatorname{PCR} 6}(\Theta)=0.1223$.

The authors conclude that "the focal element $\{\mathrm{v} 3\}$ absorbs almost all of the conflicting mass (the majority)" that we prove it is clearly untrue in the next section.

\section{REESTABLISHING THE TRUTH.}

$-+$

In order to prove that the authors' claim "the focal element $\{v 3\}$ absorbs almost all of the conflicting mass (the majority)" in [1] is wrong, we detail the calculation of the PCR6 for the three sources. First, we apply the conjunctive rule:

\begin{tabular}{llll} 
& $v_{1}$ & $v_{3}$ & $\Theta$ \\
$m_{1}$ & 0.8 & 0 & 0.2 \\
$m_{2}$ & 0.1 & 0.9 & 0 \\
$m_{3}$ & 0.4 & 0 & 0.6 \\
\hline
\end{tabular}

$m_{123 \text { conj }} \quad 0.1000 \quad 0.1080 \quad 0$

Now let's compute the total conflicting mass:

$0.8(0.9) 0.6+0.4(0.9) 0.2+0.8(0.9) 0.4=0.7920$.

This total conflicting mass, 0.7920 , was afterwards redistributed back to the elements involved in the conflict: $v_{1}, v_{3}$, and $\Theta$ proportionally respectively with their mass put in each partial conflict. There were three partial conflicts in this example. Hence:

The redistribution by PCR6 of the conflicting mass for $v_{1}$ is passing from $m_{123}\left(v_{1}\right)=0.1000$ to $m_{123 / P C R 6}\left(v_{1}\right)=0.4340$, or a gain of 0.3340 . The redistribution by PCR6 of the conflicting mass for $v_{3}$ is passing from $m_{123}\left(v_{3}\right)=0.1080$ to $m_{123 / P C R 6}\left(v_{3}\right)=0.4437$, or a gain of 0.3357 . The redistribution by PCR6 of the conflicting mass for $\Theta$ is passing from $m_{123}(\Theta)=0.0000$ to $m_{123 / P C R 6}(\Theta)=0.1223$, or a gain of 0.1223 .

Therefore $v_{3}$ actually gained from the total conflicting mass only $0.3357 / 0.7920 \approx$ $42.3864 \%$, not even half of it, not "almost all of the conflicting mass (the majority)" as the authors wrongly claim in [1]. $v_{1}$ gained close to $v 3: 0.3340 / 0.7920 \approx 42.1717 \%$, and $\Theta$ gained the difference $(15.4419 \%)$. In our opinion PCR6 did a pretty fair redistribution of the total conflicting mass. 


\section{COMMENTS ON THE PAPER "AN ALTERNATIVE COMBINATION RULE FOR EVIDENTIAL REASONING” BY SEBBAK et al., PUBLISHED IN FUSION 2014 CONFERENCE, SALAMANCA, SPAIN, JULY 2014}

Remark: If the authors applied PCR5 instead of PCR6, the total conflicting mass will be redistributed in a bigger percentage to $v_{3}$, that's why we propose to use in the applications PCR6 instead of PCR5. With MatLab, we got for PCR5 the following result: $m_{123 \mid P C R 5}\left(v_{1}\right) \approx 0.3450, m_{123 \mid P C R 5}\left(v_{3}\right) \approx 0.5327$, and $m_{123 \mid P C R 5}(\Theta) \approx 0.1223$.

Total conflict is naturally the same: 0.7920 .

The redistribution by PCR5 of the conflicting mass for $v_{1}$ is passing from $m_{123}\left(v_{1}\right)=0.1000$ to $m_{123 / P C R 5}\left(v_{1}\right)=0.3450$, or a gain of 0.2450 . The redistribution by PCR5 of the conflicting mass for $v_{3}$ is passing from $m_{123}\left(v_{3}\right)=0.1080$ to $m_{123 / P C R 5}\left(v_{3}\right)=0.5327$, or a gain of 0.4247 . The redistribution by PCR5 of the conflicting mass for $\Theta$ is passing from $m_{123}(\Theta)=0.0000$ to $m_{123 / P C R 5}(\Theta)=0.1223$, or a gain of 0.1223 .

With $P C R 5$ one gets $0.4247 / 0.7920 \approx 53.62 \%$ of the total conflicting mass redistributed to $v_{3}$, but not "almost all conflicting mass" either.

\section{ON THE CREC RULE OF COMBINATION.}

In [1], the authors proposed a new rule of combination called the CREC (Combination Rule of Evidences with Cardinalities), based on the introduction of "hidden conflict" which is mathematically problematic and doesn't make sense from the point of view of the conjunctive operator.

For example, if one considers two BBAs $m_{1}\left(\right.$.) and $m_{2}($.) and two elements $X$ and $Y$ of the power-set of a given frame of discernment, with $X$ included in $Y$, then the product mass $m_{1}(X) m_{2}(Y)$ is not entirely transferred back to $X$ with CREC rule (as it should be done in the conjunctive way), but it is split as (see example given in [1] page 4)

$m_{12}(X)=|X \cap Y| / Y \mid m_{1}(X) m_{2}(Y) \quad$ (called effective conjunctive rule)

and

$\bar{m}_{12}(X)=(1-|X \cap Y| / Y \mid) m_{1}(X) m_{2}(Y) \quad$ (called hidden conflict $)$

of course

$m_{1}(X) m_{2}(Y)=m_{12}(X)+\bar{m}_{12}(X)$,

but we disagree that $\bar{m}_{12}(\mathrm{X})$ has something to do with a "hidden conflict", and we question why such decomposition makes sense, or could be useful. 
In fact, there are many ways to make decompositions of $m_{1}(X) m_{2}(Y)$, as already proposed in Chap. 8 (An In-Depth Look at Quantitative Information Fusion Rules ) of [2], Vol. 2, pp. 205-236, or in [2], Vol. 3, Chap. 5, pp. 161-183. These books are freely available respectively on the web at http://fs.gallup.unm.edu/DSmT-book2.pdf, and http://fs.gallup.unm.edu/DSmT-book3.pdf.

For example, instead of using the Cardinality Atomicity of a focal element $X$ with respect to another focal element $Y$ in the combination fusion rules, defined by the authors as:

$c a(X \uparrow Y)=\frac{|X \cap Y|}{|Y|}$

we believe it would be more interesting of using the degree of intersection of the elements $\mathrm{X}$ and $\mathrm{Y}$, defined as:

$d(X \cap Y)=\frac{|X \cap Y|}{|X \cup Y|}$

or the degree of union of the focal elements $\mathrm{X}$ and $\mathrm{Y}$, defined as:

$d(X \cup Y)=\frac{|X \cup Y|-|X \cap Y|}{|X \cup Y|}$

or the degree on inclusion of a focal element $\mathrm{X}$ into another focal element $\mathrm{Y}$, defined as:

$d(X \subseteq Y)=\frac{|X|}{|Y|}$.

\section{CONCLUSION}

In this short note, we have reestablished the truth about the real behavior of PCR6 (and even PCR5) for Example 2 given by Sebbak et al., and we point out a problem with the mathematical definition of the "hidden conflict" introduced by the authors, which has clearly nothing to do with conflicting evidences.

\section{REFERENCES}

1. Faouzi Sebbak, Farid Benhammadi, M'hamed Mataoui, Sofiane Bouznad and Yacine Amirat, An Alternative Combination Rule for Evidential Reasoning, Proceedings of the $17^{\text {th }}$ International Conference on Information Fusion, Salamanca, Spain, 7-10 July 2014.

2. Florentin Smarandache, Jean Dezert, Advances and Applications of DSmT in Information Fusion, Am. Res. Press, Rehoboth, Vol. I, II, and III, 2004, 2006, and 2009. 